\title{
A Decentralized Passive Islanding Detection Method Based on the Variations of Estimated Droop Characteristics
}

\author{
Juan Roberto Lopez (D), Luis Ibarra*iD, Pedro Ponce (D) and Arturo Molina (DD \\ Tecnologico de Monterrey, School of Engineering and Sciences, Puente 222, Tlalpan, Mexico City 14380, Mexico; \\ A01334289@itesm.mx (J.R.L.); pedro.ponce@tec.mx (P.P.); armolina@tec.mx (A.M.) \\ * Correspondence: ibarra.luis@tec.mx
}

check for updates

Citation: Lopez, J.R.; Ibarra, L.

Ponce, P.; Molina, A. A Decentralized Passive Islanding Detection Method Based on the Variations of Estimated Droop Characteristics. Energies 2021, 14, 7759. https://doi.org/10.3390/ en14227759

Academic Editor: Javier Contreras

Received: 16 October 2021

Accepted: 29 October 2021

Published: 19 November 2021

Publisher's Note: MDPI stays neutral with regard to jurisdictional claims in published maps and institutional affiliations.

Copyright: (c) 2021 by the authors. Licensee MDPI, Basel, Switzerland. This article is an open access article distributed under the terms and conditions of the Creative Commons Attribution (CC BY) license (https:// creativecommons.org/licenses/by/ $4.0 /)$.

\begin{abstract}
A microgrid including distributed generators can operate connected to the main electrical network or in an isolated manner, referred to as island operation. The transition between both states can occur voluntarily, but a disconnection can also happen unexpectedly. The associated transients can be harmful to the grid, and compensating actions must be triggered to avoid service interruption, preserve power quality, and minimize the possibility of faults; island detection methods are essential to this end. Such techniques typically depend on communication networks or on the introduction of minor electrical disturbances to identify and broadcast unexpected islanding events. However, local energy resources are distributed, variable, and are expected to be integrated in a plug-and-play manner; then, conventional island detection strategies can be ineffective as they rely on specific infrastructure. To overcome those problems, this work proposes a straightforward, distributed island detection technique only relying on local electrical measurements, available at the output of each generating unit. The proposed method is based on the estimated power-frequency ratio, associated with the stiffness of the grid. A "stiffness change" effectively reveals island operating conditions, discards heavy load variations, and enables independent (distributed) operation. The proposal was validated through digital simulations and an experimental test-bed. Results showed that the proposed technique can effectively detect island operation at each generating unit interacting in the microgrid. Moreover, it was about three times faster than other reported techniques.
\end{abstract}

Keywords: islanding detection; microgrid; decentralized operation; distributed generation; droop characteristics

\section{Introduction}

The integration of alternative generation sources at the power grid's distribution level has led to new challenges in the control, communication, and management areas of the electrical network. Indeed, availing local resources comes with added complexity and requires coordination mechanisms to ensure their effective integration, leading to the organization of local electrical agents into microgrids. A microgrid is a cluster of distributed generation units (DGU) with power management and regulation capabilities that cover a local power demand [1,2]. Microgrids add flexibility since their operation is not restricted to a permanent connection to the main power grid; they can act independently in what is known as island operation. However, switching between operating modes implies varying dynamics and dealing with a "weaker" network whenever the main power grid is disconnected.

A microgrid interacts with the main grid through a single point of common coupling (PCC). Disconnection events can be intentional, e.g., in the case of maintenance routines, or unintentional, caused by faults or disruptive events [3]. An unintentional disconnection would force an island operating state, potentially introducing harmful transients and degrading power quality. It is then essential to detect the islanding events within the microgrid to trigger ancillary services and exert compensating actions [4]. However, DGUs are distributed and the microgrid is expected to integrate DGUs and other electrical 
agents in a plug-and-play fashion. A changing, variable, and spread microgrid poses severe challenges to a typical communications approach. Therefore, there is a need for distributed island detection algorithms.

Detection algorithms can be classified as (i) remote detection methods (RDM), (ii) passive detection methods (PDM), and (iii) active detection methods (ADM) [5]. RDMs are typically based on supervisory control techniques, relying on communication infrastructure. Then, some of their main disadvantages are communication latency and interference in powerline-based methods [6]. PDMs monitor electrical variables, e.g., at the PCC [5], to create a tripping signal according to predefined thresholds [7]. As the signal must be shared across the microgrid, PDMs typically imply the need and disadvantages of communication infrastructure. Finally, ADMs operate at individual DGUs introducing minor electrical disturbances to the grid, analyzing the response, and determining its operational state [8]. The main drawback is the degradation of power quality due to the introduced electrical disturbances and the late response depending on the electric disturbance. Also, multiple interacting DGUs may interfere with each other [9].

PDMs and ADMs are local detection techniques [10] due to the continuous monitoring and analysis of "local" electrical variables such as voltage, frequency, and active and reactive power. In PDMs, the electrical variables are constantly evaluated against an established threshold; namely, rate of change of voltage (ROCOV), rate of change of frequency (ROCOF), over/under voltage, over/under frequency, active power mismatch, and reactive power mismatch [10]. As expected, PDM techniques' performance rely on the type of acquired measurements and their location, sometimes needing additional systems to verify or broadcast the detection signal as discussed next.

For instance, Abd-Elkader et al. [11] proposed a ROCOV PDM based on detecting voltage drifts surpassing a predefined threshold. For large active power mismatches, this algorithm detects the island event $200 \mathrm{~ms}$ after the disconnection. However, small variations require the validation of a secondary strategy, implementing a physical load disconnection to raise the variation, adding $84 \mathrm{~ms}$ to the detection time. Similarly, Gupta et al. presented a ROCOF PDM [12] that uses the difference between the generated and demanded active power, the rating of the generation units, and the fundamental frequency to find the RO$\mathrm{COF}$ over a defined time window. The detection time depends on such window as the ROCOF is compared to a predefined threshold value until the sample window is closed. The algorithm also introduces small frequency deviations through the $q$-axis control of a grid-side converter. Since such disturbance can cause small periodic deviations in a "weaker" grid, they can be used as trigger signals. In the end, it detects the island condition between $100 \mathrm{~ms}$ and $200 \mathrm{~ms}$. However, the presence of externally introduced frequency drifts can cause false detection signals and a communication infrastructure is needed to gather the actual power demand.

To minimize these shortcomings, hybrid detection methods (HDM) were proposed, commonly classified as series or parallel. Series methods comprise two detection layers: a PDM or an ADM, and a high-level monitoring-based strategy. Only when the monitoring layer detects an islanding event, the PDM or ADM is activated to verify, limiting the ADM disturbances as they are only needed if the first condition is met, and minimizing false tripping. However, the detection time increases if a window-based technique is used at the second layer. A parallel configuration comprises both detection layers running concurrently, generating a tripping signal if both "agree" on a disconnection event. However, this configuration combines the weaknesses of the selected methods $[9,10]$. Indeed, the detection systems are improved by combining different strategies, but some of the advantages can be lost as well.

For example, the work presented in [9] proposes combining a PDM and an ADM in a series configuration. The ADM was placed at the second layer to minimize its polluting actions and avoid false tripping. The PDM strategy monitors the ROCOV and the rate of change of the voltage harmonic content at the PCC. A voltage index is then calculated as the negative and positive sequences ratio of the delivered voltage. Subsequently, by 
comparing the obtained voltage index to a previous voltage index value, a percentage delta can be computed. The total harmonic distortion index is then calculated by obtaining the percentile difference of the harmonic content in one fundamental signal's period. Then, the differences can be compared to specific thresholds, however, the authors do not provide further information on how the threshold values are obtained. Overall, the proposed method is capable of detecting the island event in $138 \mathrm{~ms}$ with minimum disadvantages, such as the injection of polluting action of the ADM.

Pouryekta et al. [5] presented a series RDM-PDM. Their objective was to minimize the nondetection zones (NDZ) and hasten the detection signal. The chosen RDM detected signals injected to the power-line and the PDM was ROCOV-based. It monitored the voltage phase angle during a defined time window, and yielded a detection time of $200 \mathrm{~ms}$. Nonetheless, the method relies on communication infrastructure, hindering its decentralized applicability. Finally, Bakhshi-Jafarabadi \& Popov [13] introduced an HDM based on a series PDM-ADM. The PDM is based on the ROCOV at the PCC. If a defined threshold is surpassed, the ADM verifies by distorting the reference current $d$-axis. Then, the active power is analyzed for any variation that would indicate an island operation. The entire process takes about $300 \mathrm{~ms}$ in large power mismatch scenarios; for smaller variations, the delay might be higher or the event could be missed.

Despite the advantages of HDMs, they pose future operational challenges in fully distributed microgrids. All hybrid methods include at least one detection layer bound to critical data, only available through a communication network. Therefore, methods operating in a "local" fashion such as ADM are required. Nonetheless, the polluting nature of these strategies can also have a negative effect as previously discussed. In any case, there is a need for distributed island detection methods that do not compromise the quality of the delivered energy.

Overall, DGUs are expected to operate independently within a microgrid under a distributed approach. Their management is desired to be decentralized, not relying on a communication layer or a central controller. Therefore, a passive detection algorithm is proposed in this work, operating over local DGU variables only. Specifically, the proposed algorithm analyzes the power-frequency ratio deviations at the DGU terminals without inserting disturbances to the grid. Opposed to the reviewed literature, this algorithm does not require a communication network or a secondary verifying system, interacting directly with its associated DGU and acquiring measurements directly from the DGU's outputs. Out of this, the proposal does not imply adding devices but adding a software module to the DGU controller, avoiding increased costs or maintenance.

Such a distributed approach eradicates the need for communicating a tripping signal, as each generating unit would make the same detection independently. In doing so, the cost of new communication lines can be saved, and plug-and-play features can be availed in future microgrid implementations. In addition, since the proposed method does not inject any electrical disturbance to the grid, it is possible to have multiple DGUs operating under the same island detection mechanism without major concerns regarding the interference between each other.

The proposed method was tested in simulated scenarios and experimentally through the hardware-in-the-loop approach. It exhibited a maximum detection time of $40 \mathrm{~ms}$ on a multi-inverter configuration, and of $25 \mathrm{~ms}$ for a single DGU system. Also, the proposed method was able to avoid false tripping in relevant test cases, resulting in a safe and reliable approach.

The remaining of this work is organized as follows. Section 2 discusses the different test-beds proposed across the literature to validate island detection techniques. Out of this, one test-bed is selected for testing the current proposal. Section 3 introduces the proposed detection algorithm, highlighting its operating principles. Then, Section 4 introduces a validation protocol based on the selected test-bed and the test-cases found in the literature. Sections 5 and 6 show the simulation and experimental results of this work, in more detail, 
Section 6 also describes how the experimental part of this work is carried out. Lastly, the conclusions of this work are reported in Section 7.

\section{Islanding Detection Test-Bed}

Although many island detection algorithms have been introduced over the years, they were evaluated under different configurations and test-beds, hindering a direct comparison and the evaluation of new techniques. In order to effectively locate and compare this work's proposal, the relevant literature was reviewed and three test-beds were identified:

1. Standard microgrid model. Usually, the IEEE 34-bus model is adopted.

2. Microgrid equivalent model. The structure shown in Figure 1 is used to emulate a simplified microgrid, using a three-phase parallel RLC load. Such a structure is recommended in the IEEE Std. 929 and IEEE Std. 1547 [14,15] to test islanding detection algorithms.

3. Proprietary system. A specific model that fits some desired microgrid specifications.

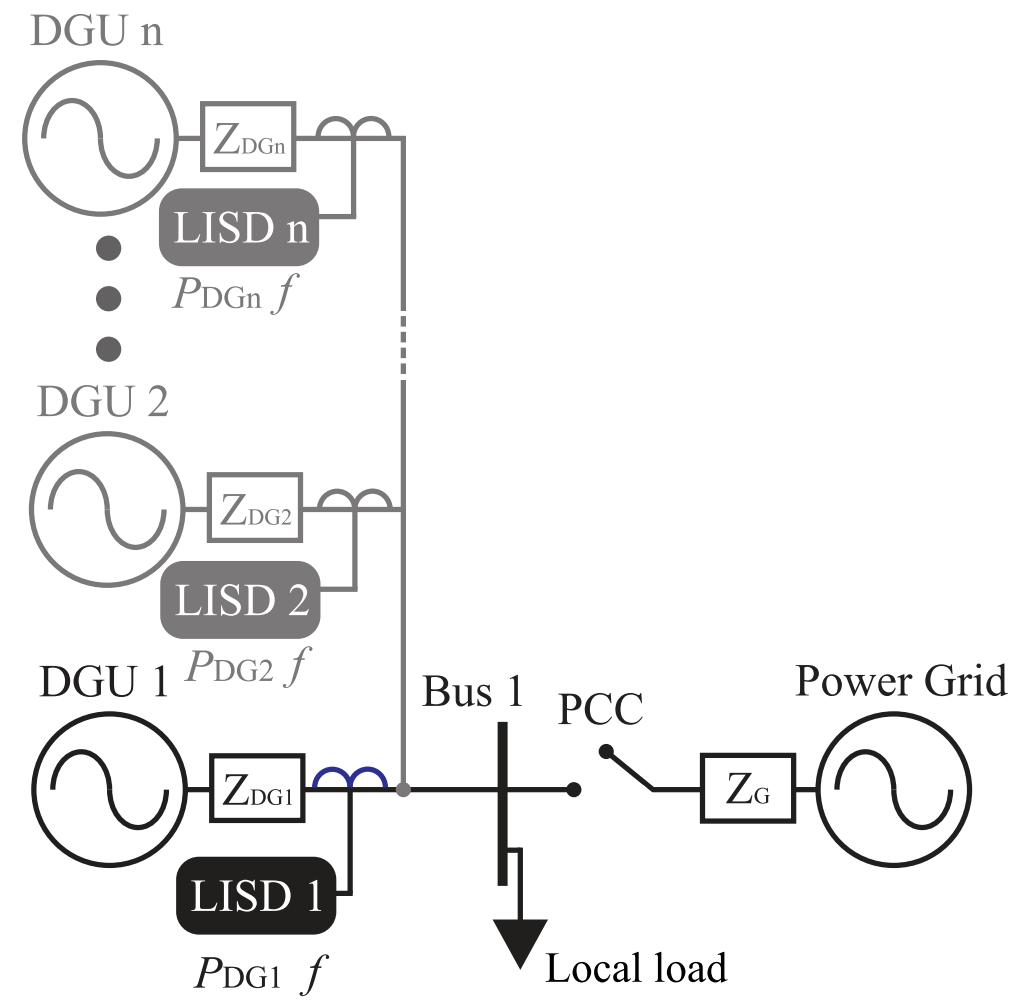

Figure 1. Basic test system schematic, from a single DGU to $n$ number of DGUs.

Table 1 enlists other reported proposals divided into the mentioned categories. As the second test-bed ( 3 phase RLC load) is being used to validate current proposals, offers the easiest implementation, and is readily replicable, it is the one considered in this work. It is important to notice that it was reported a detection time of $2.56 \mathrm{~ms}$; however, it was confirmed by simulated tests only [16]. Although that work also included an experimental section, it failed to demonstrate the same level of effectiveness and did not report any experimental detection time. Additionally, the proposed PDM was intended for intentional disconnection events. 
Table 1. State of the art review of islanding detection systems.

\begin{tabular}{|c|c|c|c|c|c|c|}
\hline Test System & $\begin{array}{c}\text { Type of } \\
\text { Detection } \\
\text { Method }\end{array}$ & $\begin{array}{c}\text { Island } \\
\text { Detection } \\
\text { Strategy }\end{array}$ & $\begin{array}{l}\text { Detection } \\
\text { Time }\end{array}$ & $\begin{array}{l}\text { Simulation } \\
\text { Validation }\end{array}$ & $\begin{array}{l}\text { Experimental } \\
\text { Validation }\end{array}$ & Reference \\
\hline \multirow{2}{*}{ IEEE 34 Bus model } & PDM & $\begin{array}{c}\text { Voltage } \\
\text { unbalance and } \\
\text { Current THD }\end{array}$ & $129 \mathrm{~ms}$ & yes & no & [17] \\
\hline & PDM & $\begin{array}{c}\text { PMU } \\
\text { monitoring }\end{array}$ & $\mathrm{N} / \mathrm{A}$ & yes & no & [18] \\
\hline \multirow{4}{*}{3 phase RLC load } & PDM & Active ROCOF & $100 \sim 200 \mathrm{~ms}$ & yes & no & [12] \\
\hline & HDM & $\begin{array}{c}\text { ROCOV, } \\
\text { Voltage THD, } \\
\text { and reactive } \\
\text { power } \\
\text { mismatch }\end{array}$ & $138 \mathrm{~ms}$ & yes & yes & [9] \\
\hline & $\mathrm{ADM}$ & $\begin{array}{c}\text { Frequency } \\
\text { drifts }\end{array}$ & $140 \mathrm{~ms}$ & yes & yes & [19] \\
\hline & PDM & $\begin{array}{c}\text { Voltage and } \\
\text { frequency drifts }\end{array}$ & $2.56 \mathrm{~ms}$ & yes & no & [16] \\
\hline \multirow{4}{*}{ Proprietary system } & HDM & $\begin{array}{c}\text { Voltage } \\
\text { Threshold and } \\
\text { MPPT } \\
\text { disturbance }\end{array}$ & $300 \mathrm{~ms}$ & yes & yes & [20] \\
\hline & PDM & ROCOV & $200 \sim 300 \mathrm{~ms}$ & yes & no & [11] \\
\hline & HDM & $\begin{array}{c}\text { Power line } \\
\text { signal and } \\
\text { Voltage phase }\end{array}$ & $200 \mathrm{~ms}$ & yes & yes & [5] \\
\hline & HDM & $\begin{array}{l}\text { ROCOV } \\
\text { and d-axis } \\
\text { current } \\
\text { disturbance }\end{array}$ & $300 \mathrm{~ms}$ & yes & no & [13] \\
\hline
\end{tabular}

\subsection{The 3-Phase RLC Load Testbed}

As commented above, such a test system is not only used by other works in the literature but is also recommended for islanding detection tests by the IEEE Std. 929 and IEEE Std. 1547 [14,15]. It comprises one or many DGUs connected to a single bus feeding a three-phase parallel RLC load. Each enabled DGU comprises a three-phase two-level voltage source inverter (VSI) operating under constant-power conditions [9].

Due to the easy implementation and high flexibility of the described test systems, some secondary tests become available to guarantee a safe performance. Other electrical agents can add disturbances coming from large load switching, nonlinear load integration, and voltage dips on the power grid side. Those disturbances are prone to cause wrong triggering of the island detection technique, enabling the testing of false positives. Such secondary tests are described later in Section 4.

\subsection{Constant Power Approach for DGU Control}

Since the interfaced DGUs are expected to be operating under a grid-connected scheme prior to disconnection, a constant power approach is used to control all DGUs as done in [9]. This control strategy aims to deliver a constant amount of active and reactive power ( $P$ and $Q$, respectively). It comprises a cascaded control loop that computes the necessary 
inverter's outputs to uphold the measured power to given set-points, i.e., $P^{*}$ and $Q^{*}$ (see Figure 2). The instantaneous active and reactive powers are computed through

$$
\hat{P}=\mathbf{V}_{d q}^{\top} \mathbf{I}_{d q}, \quad \hat{Q}=\mathbf{V}_{d q}^{\top}\left[\begin{array}{cc}
0 & -1 \\
1 & 0
\end{array}\right] \mathbf{I}_{d q}
$$

where $\mathbf{V}_{d q}=\mathbf{T V}_{\mathrm{abc}}$ and $\mathbf{I}_{d q}=\mathbf{T I}_{\mathrm{abc}}$, being $\mathbf{V}_{\mathrm{abc}}$ and $\mathbf{I}_{\mathrm{abc}}$ the three-phase voltages and currents sensed at the DGU's output, and T the Park transformation aligned to the $\alpha$ axis.

$$
\mathbf{T}=\sqrt{\frac{2}{3}}\left[\begin{array}{ccc}
\cos (\hat{\theta}) & \cos \left(\hat{\theta}-\frac{2 \pi}{3}\right) & \cos \left(\hat{\theta}+\frac{2 \pi}{3}\right) \\
-\sin (\hat{\theta}) & -\sin \left(\hat{\theta}-\frac{2 \pi}{3}\right) & -\sin \left(\hat{\theta}+\frac{2 \pi}{3}\right)
\end{array}\right] .
$$

In addition, a three-phase phase-locked loop (PLL) is used to track the voltage's frequency $f$ and phase $\theta$ at the DGU's output (a MAF-PLL was used in this work [21]). The estimated phase $\hat{\theta}$ is used as an input to (2), whereas the estimated frequency $\hat{f}$ and the previously computed $\hat{P}$ are fed to the proposed local island detection algorithm (LISD).

The control loop comprises a parallel PID arrangement that processes the $d$ and $q$ components individually, first using the power error to compute current set-points, $i_{d}^{*}$ and $i_{q}^{*}$, and then the current error to output the required inverter's voltage, i.e., $\mathbf{V}_{d q}^{*}=\left[\begin{array}{ll}v_{d}^{*} & v_{q}^{*}\end{array}\right]^{\top}$. In this work, a 3-phase 2-level inverter was considered together with a space-vector PWM technique. To provide the three-phase input required by the switching technique, $\mathbf{V}_{\mathrm{abc}}^{*}=T^{-1} \mathbf{V}_{d q}^{*}$ was computed as shown in Figure 2. Finally, the PID controllers were tuned by trial and error (a customary practice) with the help of the simulated test-bed. The reader is referred to [9] where the described controller is presented in depth.

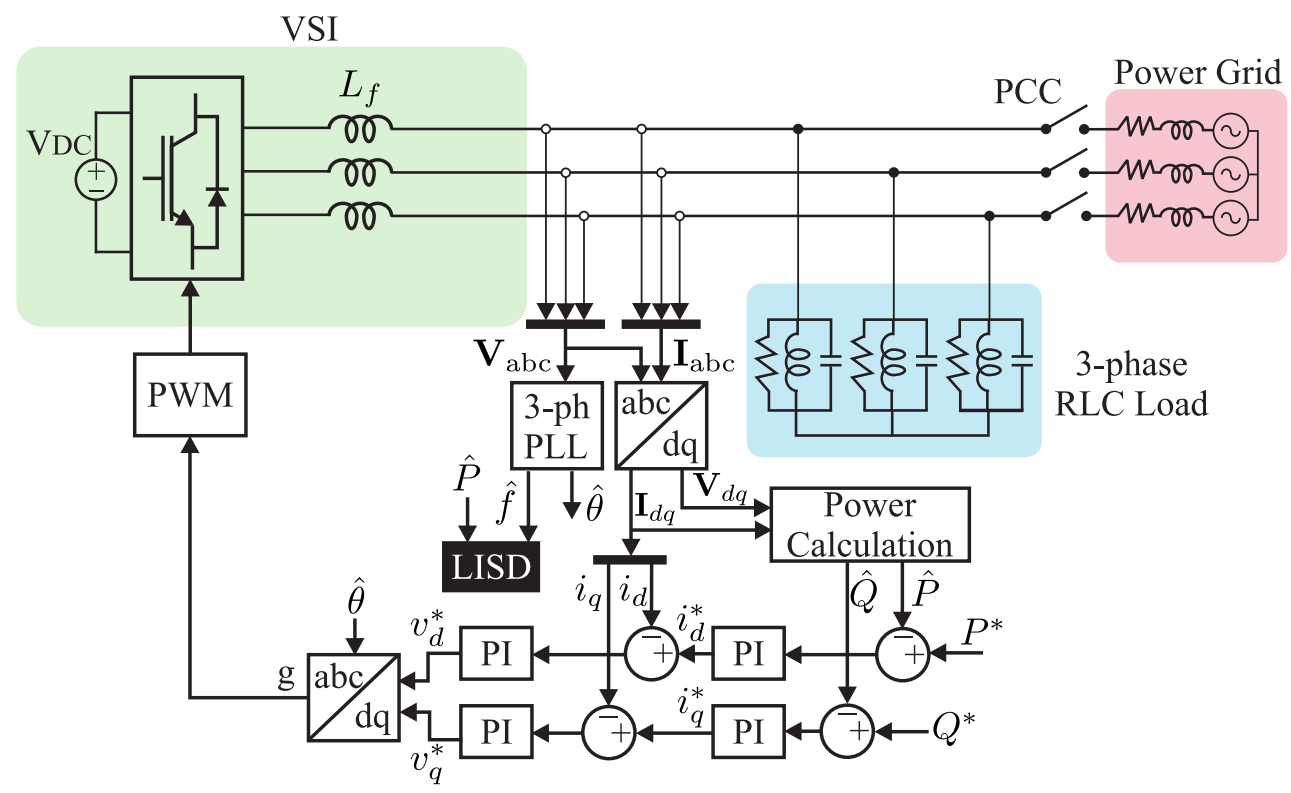

Figure 2. Control block scheme for interfaced DGU and proposed LISD.

\section{Island Detection Algorithm Design}

The algorithm is based on the power-frequency drooping characteristics of the generating elements the microgrid takes its power from. Drooping is a natural phenomenon associated with turbines' loading at conventional power plants, and alternative sources commonly use the virtual synchronous generator approach, where the drooping characteristics are imposed by design, depending on the DGU rating [22]. It mainly implies that the generating source will degrade its rotating speed as it becomes electrically loaded, exhibiting a linear, inverse relationship between the taken active power and the output frequency.

The main grid is stiff as it is supported by multiple, massive generators, implying that its drooping will not be noticeable because the connected loads (and DGUs) are always 
relatively small. Then, it is expected that in grid-connected operation, the microgrid behaves like a stiff network for which power variations do not affect the main frequency. Nonetheless, in island operation, the microgrid relies on limited DGUs comprising a "weaker" grid with clear drooping characteristics. Then, the stiffness of a microgrid is variable as it transitions between connected and islanded states, showing different droop characteristics (slopes) as illustrated in Figure 3. This would indicate that a change in the estimated slope can be used as a local islanding detection event, eradicating the need for a communication infrastructure or the introduction of disturbances by ADMs.

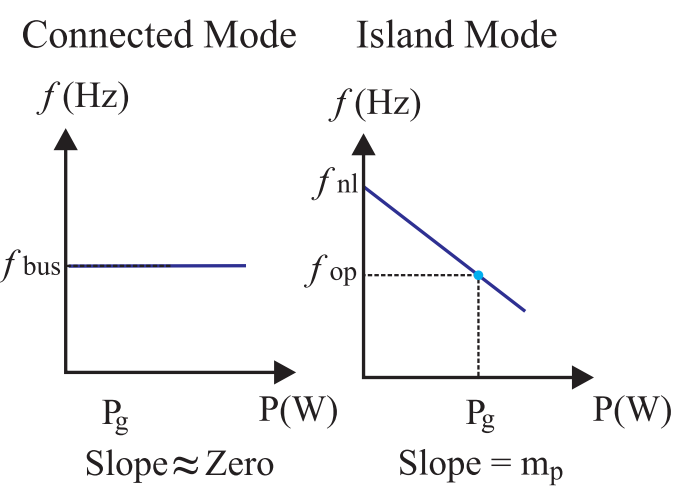

Figure 3. Islanding detection through droop control characteristics.

In more detail, the droop slope $m_{p}$ correlates the delivered active power $P_{g}$ and the operating frequency $f_{\mathrm{op}}$ linearly. The resulting line crosses the vertical axis at $f_{\mathrm{nl}}$, the noload point, where the delivered active power is zero. This point is defined as the maximum allowed frequency of the system. If $m_{p} \approx 0$, the operating frequency is that of the main grid; then, $f_{\text {op }}=f_{\text {bus }} \forall P_{g}$.

The proposed LISD monitors local electrical variables to find the said linear approximation, i.e.,

$$
f_{\mathrm{op}}=m_{p} P_{g}+f_{\mathrm{nl}} \text {. }
$$

A shift-register is populated with input sampled data pairs $(\hat{f}[k], \hat{P}[k])$, where $k \in \mathbb{N}$ represents the $k$ th sample of the signals, clocked at a given sample time $T_{s}$. Only $n \in \mathbb{N}$ samples are stored, then, one can build the vectors

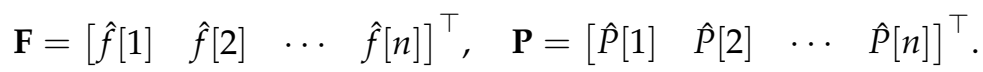

The line parameters are then estimated by

$$
\left[\begin{array}{c}
\hat{m}_{p}[k] \\
\hat{f}_{\mathrm{nl}}[k]
\end{array}\right]=\left(\mathbf{F}^{\prime \top} \mathbf{F}^{\prime}\right)^{-1}\left(\mathbf{F}^{\prime \top} \mathbf{P}\right)
$$

where $\mathbf{F}^{\prime}=[\mathbf{F} \mid \mathbf{1}]$, i.e., a column with ones was appended to $\mathbf{F}$.

The line estimator includes a mechanism to discard incorrect or inessential estimates. If no operating changes occur in the grid and $\mathbf{F}$ and $\mathbf{P}$ hold the same value $n$ times, Equation (5) will diverge and output non-numerical data. Programming languages are capable of detecting such issues (normally using a not-a-number value). Therefore, if divergence is detected, the estimated values are forced to

$$
\hat{m}_{p}[k]:=\hat{m}_{p}[k-1], \quad \hat{f}_{\mathrm{nl}}[k]:=\hat{f}_{\mathrm{nl}}[k-1] .
$$

Initialization is also needed, i.e., $\hat{m}_{p}[1]$ and $\hat{f}_{\mathrm{nl}}[1]$ must hold a value provided beforehand. Finally, a frequency estimate $\hat{f}^{\prime}[k]$ is computed using (3) with $\hat{m}_{p}[k]$ and $\hat{f}_{\mathrm{nl}}[k]$, and the estimation error

$$
e=\left(\hat{f}[k]-\hat{f}^{\prime}[k]\right)^{2}
$$


is used to detect relevant changes, i.e., operating conditions incompatible with the estimated droop characteristic. The regression algorithm is only enabled if $e>e^{*}$, a threshold constant decided by the designer. Notice that during island operation, $m_{p}$ would depend on the DGUs stiffness whereas the grid's operating point $(P, f)$ on the loads. Then, load variations still represented by (3) do not imply a change in $m_{p}$. The complete estimator is shown in Figure 4.

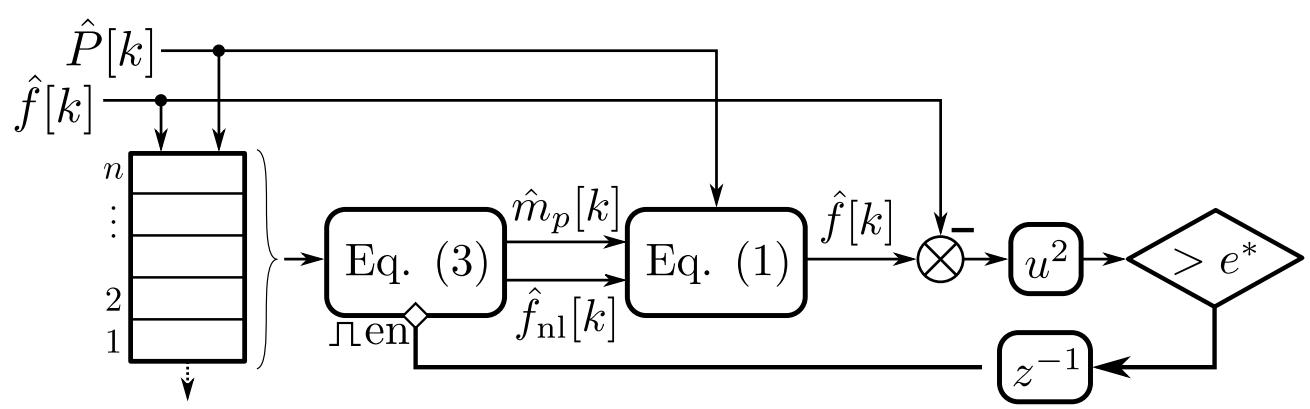

Figure 4. Block diagram of the proposed linear regression algorithm.

In order to detect the islanding condition, the LISD computes the relative variation $\Delta \hat{m}_{p}$ between estimated slopes separated by $\tau$ samples (8).

$$
\Delta \hat{m}_{p}=\left|\frac{\hat{m}_{p}[k]-\hat{m}_{p}[k-\tau]}{\hat{m}_{p}[k]}\right| .
$$

Clearly, if $\Delta \hat{m}_{p}>\Delta \hat{m}_{p}^{*}$, a threshold constant set beforehand, the LISD triggers an islanding signal. It is possible for the system to vary $m_{p}$, mostly due to changes in DGUs operation. Then, $\Delta \hat{m}_{p}^{*}$ should consider an admissible interval representing island scenarios. The entire algorithm is represented in Figure 5.

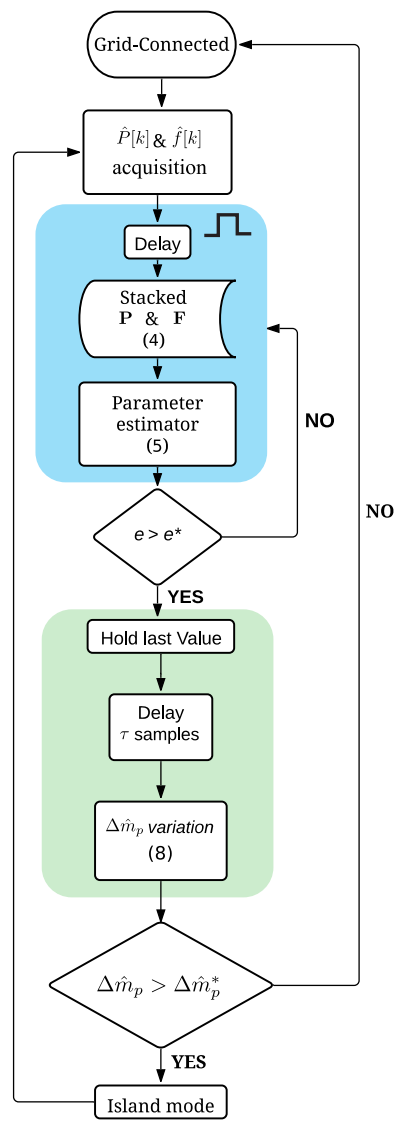

Figure 5. Flow diagram of the proposed local islanding detection algorithm. 
The performance of the proposed algorithm is constrained to the algorithm's resolution given by $T_{s}, n$, and $\tau$. By increasing $T_{s}$ the linear regression improves; however, the detection time significantly increases. On the other hand, the detecting time can be improved with a low $\tau$; nonetheless, it could also create false triggering signals. In the end, there is a trade-off between detection time and regression precision. Also, the quadratic error threshold plays an important role in the amount of data that is further processed. These considerations are discussed in the sequel.

\section{Validation Protocol}

Performance tests are required to verify the correct operation of the islanding detection algorithm, specially in scenarios that could cause false or real triggering signals. For instance, dynamic changes within a connected microgrid must not trigger an islanding event. Similarly, a disconnection must trigger it within $2 \mathrm{~s}$ according to the IEEE standard 1547 [14]. Finally, to cope with current and expected microgrid operating conditions, multiple DGUs (multi-inverter configuration) must be tested together.

The reported testbeds based on an interfaced three-phase RLC load (see Table 1) validate their proposals differently. For example, the work reported in [12] presents load switching tests, including resistive, inductive, and capacitive load switching scenarios. Similarly, the work presented in [19] test different quality factors on the interfaced RLC load. In contrast with these proposals, the work reported in [16] conducts a validation test addressing the dynamics of two interfaced DGUs, feeding a single three-phase RLC load; however, it did not analyze switching load conditions. Lastly, the work presented in [9] considered the dynamics of load switching scenarios, grid faults, and the interaction of multiple DGUs. Despite the differences, all reported test-beds comprise one or multiple DGUs feeding a single three-phase RLC load in a grid-connected configuration as showed in Figure 2.

In an attempt to aggregate the reported test conditions, the proposed test protocol consists of five different cases [9]:

- $\quad$ Case 1: Simple disconnection event with a single DGU.

- $\quad$ Case 2: Large load integration in grid-connected mode.

- $\quad$ Case 3: Non-linear load integration in grid-connected mode.

- Case 4: Voltage dip on the supply system.

- $\quad$ Case 5: Simple disconnection event in a multi-inverter system.

The details about each case study are analyzed next so that the detection time and false island detection results can be compared under fair conditions. As the test-bed and its parameters are those used in [9], the obtained results would be directly comparable to that work. Moreover, the results in [9] were proven superior to another typical detection approach proposed in [23], making the comparison twofold. As the used test-bed is the one recommended by the IEEE Std. 929 and IEEE Std. 1547 [14,15], the present results could be compared in the future with new proposals.

\subsection{Case 1: Simple Disconnection Event with a Single DGU}

The disconnection occurs at a specific time during the test. By opening the PCC, the single interfaced DGU assumes the task of feeding the connected RLC load. For this disconnection scenario, it is expected that the tested LISD triggers an islanding signal within the $2 \mathrm{~s}$ according to the IEEE standard 1547 [14].

\subsection{Cases 2 and 3: Load Integration in Grid-Connected Mode}

The incorporation of new loads, especially large and nonlinear loads, can cause small frequency ripples when connected to the microgrid, even in a grid-connected configuration. Those ripples may trigger a false islanding signal.

In more detail, case 2 integrates a large load equal to half of the original power demand, while case 3 integrates a three-phase rectifier feeding a load equal to half of the supplying power demand (nonlinear load integration). Both tests integrate their corresponding load 
at a specific instant. It is the objective of this case to evaluate if the proposed algorithm can avoid producing false tripping signals due to the caused frequency ripples.

It is important to highlight that in island detection strategies that require some comparison against a specific threshold, false tripping is possible during load variations. In the context of the proposed LISD, the frequency ripple created due to the load integration leads to variations in the estimated droop characteristic of the generator. Then, false tripping could occur if $\Delta \hat{m}_{p}^{*}$ is set incorrectly. Such threshold can be obtained by executing this test and obtaining the maximum variation of $\Delta \hat{m}_{p}$.

\subsection{Case 4: Voltage Dip on the Supply System}

Case 4 treats with the effect of any grid faults that do not cause an islanding event, a common fault is that of low-magnitude, sudden voltage dips, affecting the delivered voltage with little to no effect on the delivered frequency. For that matter, any islanding algorithm should be capable of avoiding false tripping during voltage dips.

\subsection{Case 5: Simple Disconnection Event in a Multi Inverter System}

The presence of multiple DGUs can significantly change the dynamics of a microgrid. During islanding events, all DGUs must trip to sustain a reliable operation during and after the transition event. Due to communication dependencies, any islanding event signal can be delayed or even lost in large microgrids. As the LISD proposal is decentralized, this case tests if many interacting DGUs are capable of tripping, considering that communication does not exist among them. A four-DGU system is tested to evaluate the performance of the proposed LISD in a multi-inverter arrangement. Each inverter is equipped with its own LISD.

\section{Simulation Results}

All the described simulation cases are carried out using the Specialized Power Systems toolbox of MATLAB Simulink, using the parameters listed in Table 2. Notice that, as depicted in Figure 2, the load considered is a parallel RLC load having its resonance frequency $1 / \sqrt{L C}$ matching that of the local utility operating frequency. This is assumed to be the worst-case scenario for successful detection of unintentional islanding [9]. The voltage's amplitude, frequency, and the variations of $\Delta \hat{m}_{p}$ were acquired and plotted. In the simulation, $\Delta \hat{m}_{p}$ is not latched when the island condition is detected, allowing the visualization of $\Delta \hat{m}_{p}$ changes after the disconnection event and during island operation. All simulated tests were programmed to disconnect from the main grid or modify the existing loads at $t=1.4 \mathrm{~s}$ after the system reached steady state.

The $\Delta \hat{m}_{p}^{*}$ threshold was set to a constant value (35\% as will be later explained) for all simulation results. As described in Section 4, it can be obtained by executing the test related to case 2. A more in-depth description is given in the results below. It is essential to notice that the proposed LISD is not constrained to operate in some load range and that the installed load value is not necessary for the LISD operation. As this algorithm evaluates the grid's stiffness, $\Delta \hat{m}_{p}$ would remain a valid metric in different load ranges as long as the island and grid-connected operation regimes exhibit different power-frequency slopes. 
Table 2. Simulation and system parameters.

\begin{tabular}{cccc}
\hline Parameter & Value & Parameter & Value \\
\hline$T_{s}$ & $1 \mathrm{~ms}$ & Load resistance & $26.45 \Omega$ \\
$e^{*}$ & 0.01 & Load inductance & $23.109 \mathrm{mH}$ \\
$\Delta \hat{m}_{p}^{*}$ & 0.35 & Load capacitance & $0.438 \mathrm{mF}$ \\
$n$ & 10 & Grid Resitance & $0.01 \Omega$ \\
$\tau$ & 10 & Grid inductance & $0.3 \mathrm{mH}$ \\
$V_{a b c}$ & $460 \mathrm{~V}_{\mathrm{RMS}}$ & ZDGU1 & $0.012+j 0.0052 \Omega$ \\
$f$ & $50 \mathrm{~Hz}$ & ZDGU2 & $0.015+j 0.0053 \Omega$ \\
$V_{D C}$ & $800 \mathrm{~V}$ & ZDGU3 & $0.019+j 0.0055 \Omega$ \\
$L_{f}$ & $3.8 \mathrm{mH}$ & ZDGU4 & $0.019+j 0.0055 \Omega$ \\
Rated power & $8 \mathrm{~kW}$ & & \\
\hline
\end{tabular}

\subsection{Case 1: Islanding Detection with a Single DGU}

The first simulation scenario consisted of a simple disconnection event. The microgrid was disconnected at $1.4 \mathrm{~s}$ from the main power grid, leaving all power-feeding requirements to the DGU. The performance of the LISD is shown in Figure 6. The disconnection event clearly affected the system's frequency and the proposed island detection algorithm was triggered at 1.414 s, i.e., with a delay of $14 \mathrm{~ms}$. It can be seen that further frequency variations would have again triggered the detection algorithm. However, one can latch the first tripping signal and exert the compensation mechanisms from that point on.


Figure 6. Case 1: Island detection test to evaluate detection delay.

\subsection{Cases 2 \& 3: Load Integration}

The system's load was first increased by $50 \%$ according to the parameters in Table 2 . As shown in Figure 7, the $\Delta \hat{m}_{p}$ variations caused by the large load integration raised to $30 \%$. Then, to avoid false tripping signals caused by load variations, the threshold value $\Delta \hat{m}_{p}^{*}$ must be above $30 \%$. Out of this, $\Delta \hat{m}_{p}^{*}$ was set to $35 \%$ as illustrated in the presented results.

As discussed above, it is recommended to first analyze the grid to find its usual $m_{p}$ and weigh load variations to find admissible $\Delta \hat{m}_{p}$ values. Instead, and as done in this work, the threshold can be set after running simulations considering critical variations. It is essential to consider that $\Delta \hat{m}_{p}^{*}$ would vary depending on $n, \tau, T_{s}$, and the grid parameters; 
as a result, it would be difficult to derive it analytically. In this case, the simulated approach was preferred.
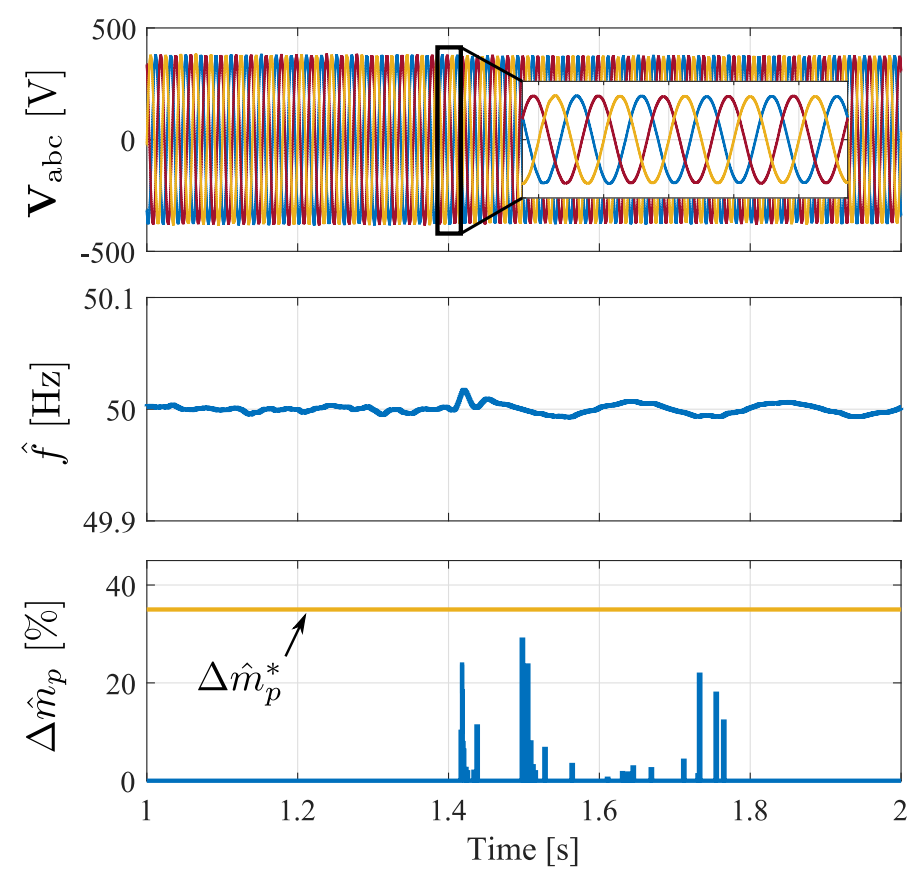

Figure 7. Case 2: Large switching load test to discard false tripping.

Similarly, case 3 considered the integration of a nonlinear load, and its results are shown in Figure 8. The nonlinearity of the rectifier exhibited a negligible effect on the performance of the proposed algorithm. In this case, the estimated droop characteristic does not show a significant jump in the estimated slope change $\Delta \hat{m}_{p}$ that could trigger any false islanding signals. Thus, neither of the tested scenarios that dealt with the integration of a large load or nonlinear load triggered a false positive signal during these tests.
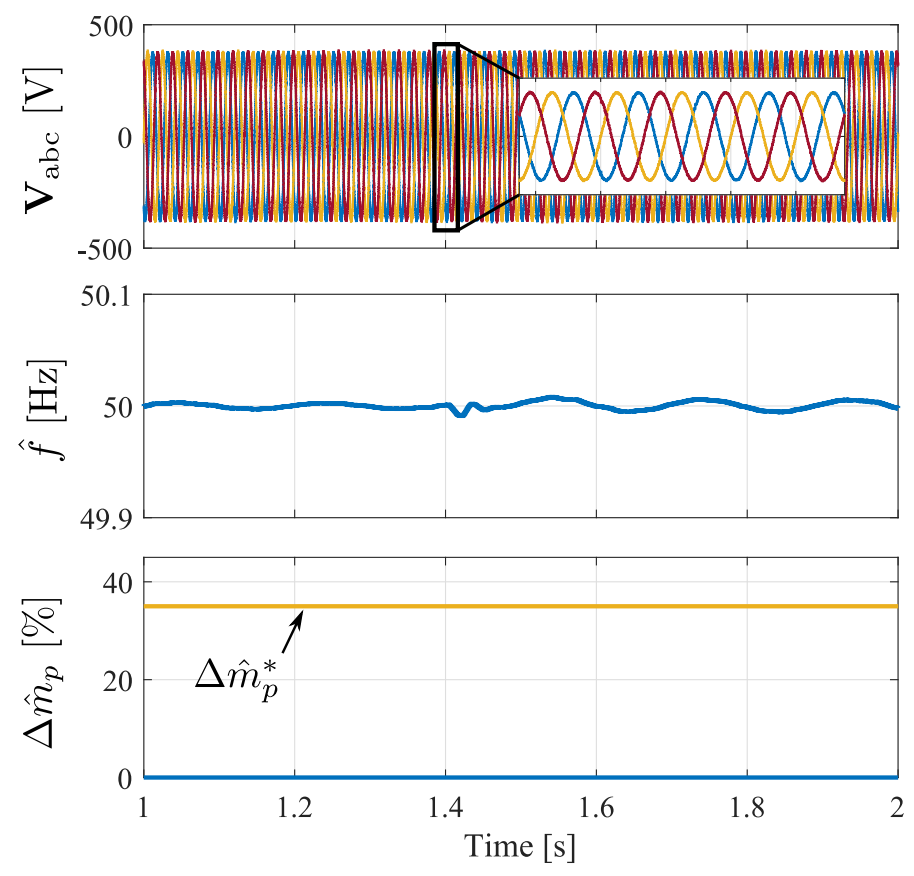

Figure 8. Case 3: Nonlinear load integration test to discard false tripping. 


\subsection{Cases 4: Voltage Dip}

Figure 9 shows the performance of the LISD after a voltage dip of 0.13 p.u. occurred (as tested in [9]). As shown, the estimated frequency did not change drastically; therefore, there is no change in the estimated droop characteristics of the DGU. As the proposed algorithm deals with frequency and power variations and not directly with the voltage levels, the LISD did not trigger a false islanding signal, exhibiting reliability in the case of low magnitude grid faults. Recall that $\hat{m}_{p}[k]$ only changes if the variation is "relevant." Then, even in the presence of frequency variations, $\Delta \hat{m}_{p}$ would not necessarily change if $e^{*}$ is not surpassed as discussed around (7).



Figure 9. Case 4: Voltage dip test to discard false tripping.

\subsection{Cases 5: Island Detection in Multiple Inverter System}

Figure 10 shows the result of the islanding test in the multiple inverter configuration. The proposed LISD algorithm was equipped locally at each inverter system (four in total), and each was capable of detecting the islanding event by calculating an individual $m_{p}$ using the variables locally available at the DGUs outputs. The islanding signal was triggered at $1.412 \mathrm{~ms}$, exhibiting a $12 \mathrm{~ms}$ delay after disconnection. The performance of the LIDS was slightly faster in a multiple invert system due to the added variations by other inverter systems in the microgrid. It is important to mention that, the impedance values corresponding to each of the DGU are taken from [24] as the authors suggest such values to conduct validation tests in a multi-inverter configuration.

Indeed, the correct performance of any islanding detection algorithms is crucial in a multiple DGU system due to the different dynamics of each contributing agent. It is also important that no counteraction occurs between DGUs, as in the case of ADMs that can introduce electrical disturbances that can have an effect on other electrical agents connected to the microgrid. Thus, the proposed algorithm detected the disconnection locally, without relying on communication lines nor introducing other electrical disturbances into the microgrid's main bus. The LISD is a noninvasive implementation that can be attached to the DGU. Nonetheless, the threshold value adjusted in case 2 needs to be properly set to avoid any false tripping signals in such conditions. 

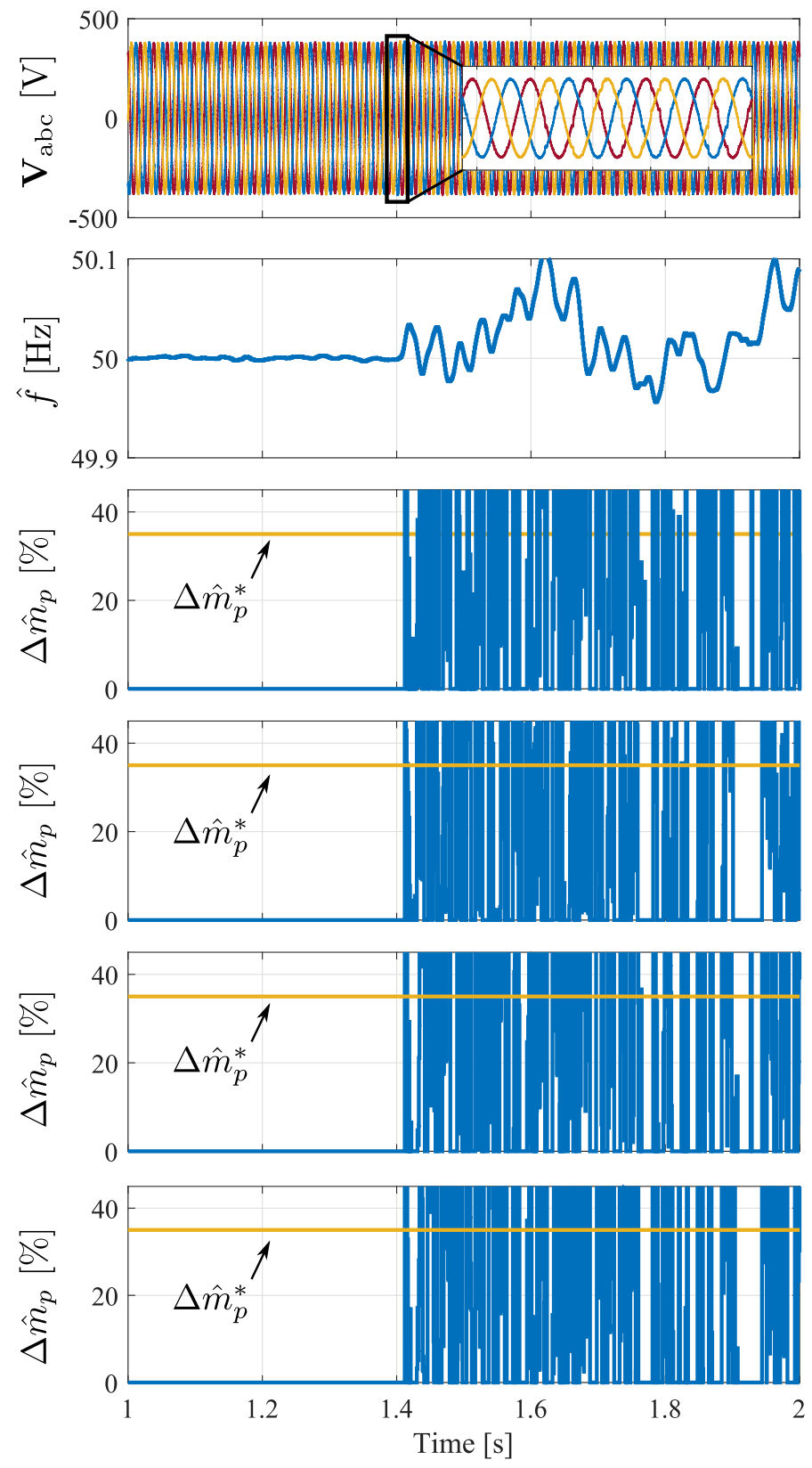

Figure 10. Case 5: Multiple DGUs test to check non-interference among DGUs tripping.

\section{Experimental Validation}

The experimental component in this work consists of hardware in the loop (HIL) realtime (RT) simulation test-bed. Two RT systems are interconnected via their analog I/Os. The first RT simulator is an OP5700 from OPAL technologies [25], this simulator runs and controls the system described in Figure 1 but not the LISD blocks. The second RT simulator is a Boom-Box by Imperix [26], ideal for rapid control prototyping and validation of diverse power electronic applications. This simulator is in charge of running the proposed LISD algorithms separately. The relevant signals are acquired with an oscilloscope.

The OP5700 is programmed to have a user-controlled switching signal to dis/connect the main grid at the PCC, or to trigger the loads' integration. This switching signal also triggers the oscilloscope using a digital output, collecting three-phase voltages and islanding signals. The primary control of the DGU is fed in a loop-back configuration through the multiple analog I/O ports of the OP5700. The active power and frequency signals are also taken from the analog output ports and connected to an Imperix proprietary interface, operating as the communication bridge between both systems. Finally, the 
OP5700 is programmed to run at $50 \mu$ step time, i.e., the solver timestep (not to be confused with $T_{S}$, which is managed at the Boom-box).

On the other end of the experimental validation, the Imperix Boom-Box receives the active power and frequency signals required for the operation of the proposed LISD. The proposed algorithm runs at a step time of $T_{S}=1 \mathrm{~ms}$ and outputs the islanding signal if such conditions are detected. This signal is carried out through a single analog output port and fed to the oscilloscope for precisely finding the delay time. Figure 11 shows the experimental configuration and connections between RT time simulation platforms.

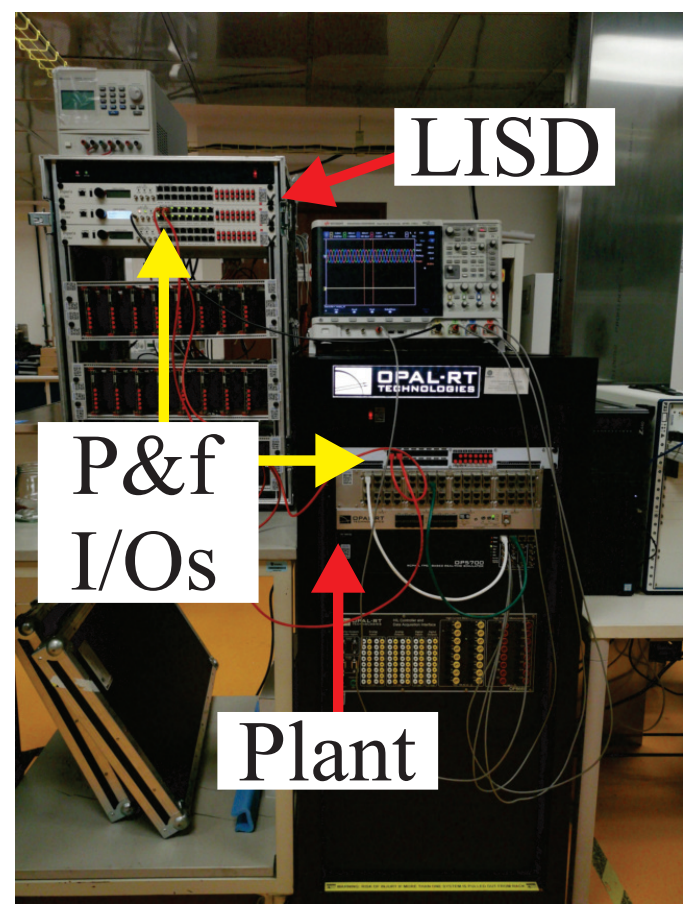

Figure 11. Real-Time Experimental Test-bed.

Case 1 was carried out as the first experimental validation test, consisting of a triggered disconnection. The Boom-Box RT system did output the islanding signal exactly $25 \mathrm{~ms}$ after the disconnection event as shown in Figure 12. Since the triggering signal occurs at $t=0$, a manual offset was used to align the rising islanding signal to the horizontal center of the oscilloscope, then obtaining the delay time as the horizontal offset. Subsequently, the load integration tests were performed in the same manner, again acquiring the islanding signal coming from the Boom-Box RT simulator. In both cases, the integration of an external load did not trigger a false islanding signal as shown in Figures 13 and 14 (three-phase parallel load and nonlinear load integration, respectively). This indicates that the proposed LISD algorithm can withstand large load switching events without tripping.

Lastly, a multi-inverter system was tested, consisting of four voltage synchronous inverters operating on a constant power control scheme while feeding a $32 \mathrm{~kW}$ load (the rated power was multiplied by the number of DGUs). Each inverter is tasked to deliver a constant power output of $8 \mathrm{~kW}$, and their output impedances are set to be different to represent different locations of DGUs (see Table 2). In this case, only the active power and frequency values of three DGUs were fed into the BoomBox due to the availability of I/O ports; therefore, only the islanding signal of these particular DGUs is graphically shown. 


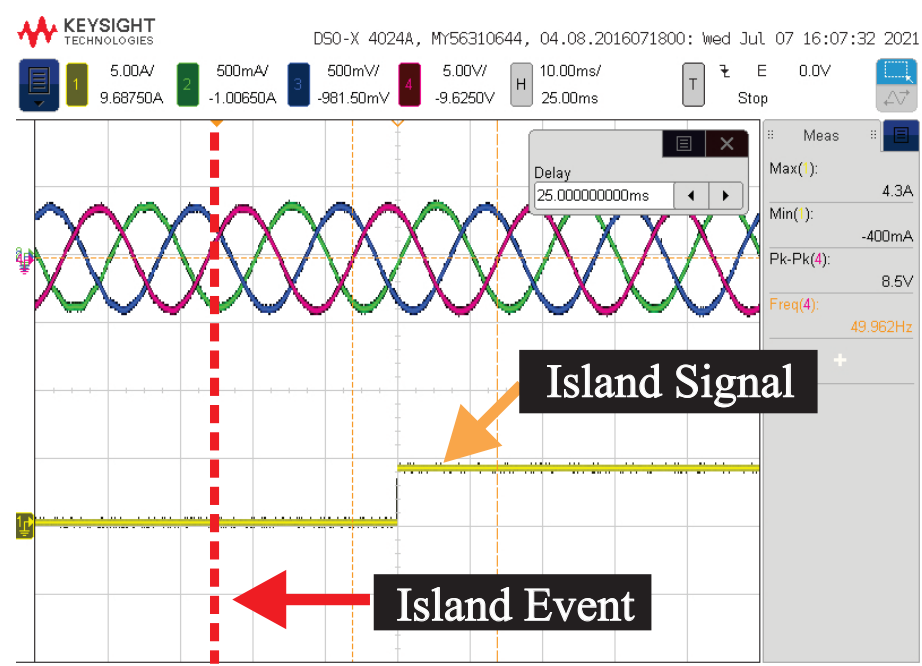

Figure 12. Single load islanding experimental results, detection time: $25 \mathrm{~ms}$.

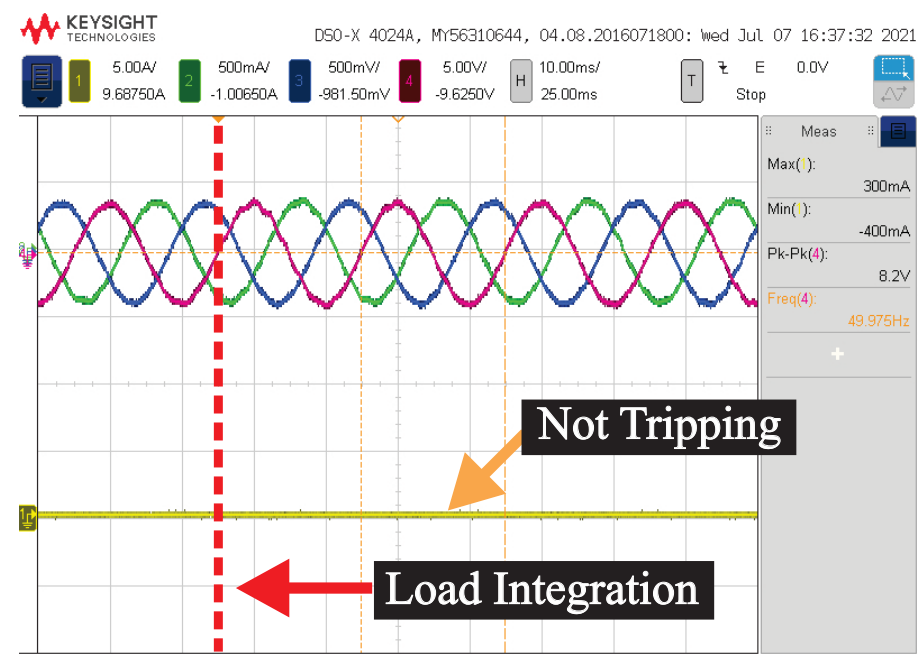

Figure 13. Large load integration tripping experimental results.

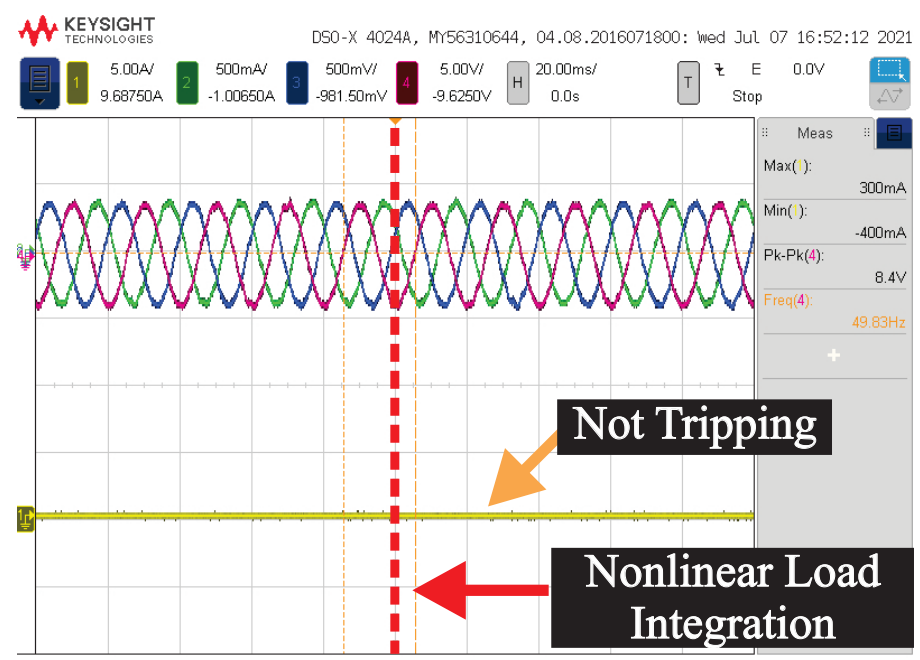

Figure 14. Nonlinear load integration tripping experimental results.

The four islanding signals were triggered after $27 \mathrm{~ms}$ (DGU1), $32 \mathrm{~ms}$ (DGU2), $40 \mathrm{~ms}$ (DGU3), and $40 \mathrm{~ms}$ (DGU4). The acquired signals from the first three DGUs are shown in 
Figure 15. It is noteworthy that the delay time seems to be associated with the distance from the PCC to the DGU, and that the simulated unit (DGU4) yielded the same delay as DGU3, both sharing the same output impedance. Each of the islanding signals was generated by monitoring only the local power and frequency measurements of each inverter. These results indicate that each DGU is capable of identifying the disconnection event even in a multiple inverter configuration without external communications.

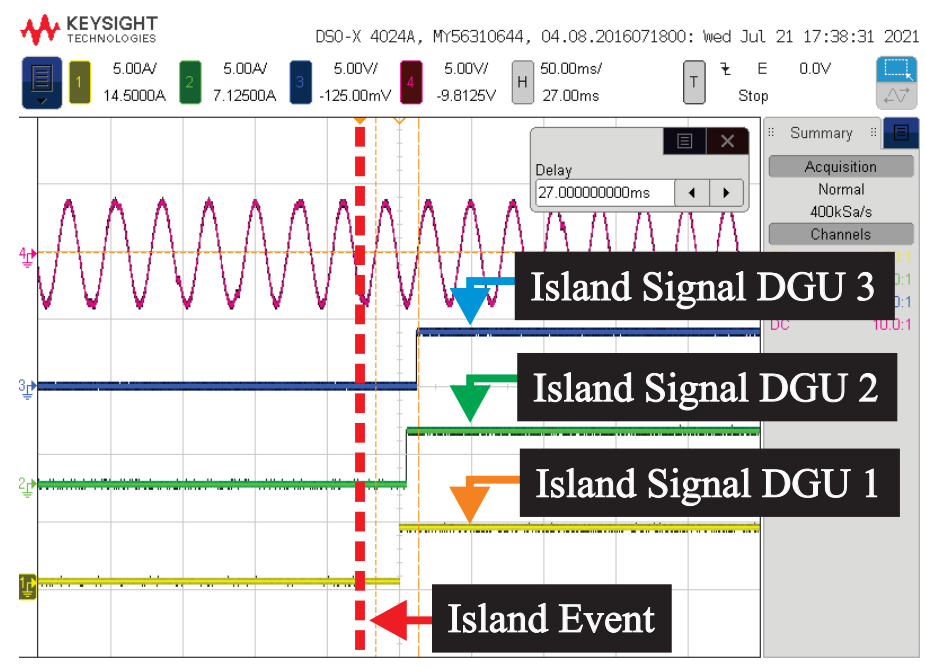

Figure 15. Multiple inverter island experimental results, detection time: (i) DGU1 27 ms; (ii) DGU2 32 ms; (iii) DGU3 40 ms.

Table 3 shows a summary of the reported results compared to the work presented in [9], addressing a hybrid islanding detection algorithm based on voltage unbalances and total harmonic distortion. This comparison is relevant because the authors have compared their proposal to other hybrid detection methods and tested their islanding algorithm under the same simulation scenarios presented in Section 4. Therefore, the LISD was validated as an improved detection mechanism over previously presented methods.

Table 3. Performance comparison of Islanding detection algorithms.

\begin{tabular}{ccccccc}
\hline \multirow{2}{*}{ ISD } & $\begin{array}{c}\text { Simulation } \\
\text { Environment }\end{array}$ & Case 1 & Case 2 & Case 3 & Case 4 & Case 5 \\
\hline \multirow{2}{*}{ LISD } & Off-line & $14 \mathrm{~ms}$ & Not tripping & Not tripping & Not tripping & $12 \mathrm{~ms}$ \\
\cline { 2 - 7 } & Real-Time & $25 \mathrm{~ms}$ & Not tripping & Not tripping & Not tripping & $27 \mathrm{~ms} 30 \mathrm{~ms}$ \\
$40 \mathrm{~ms}$ & Not tripping & Not tripping & $110 \mathrm{~ms}$ \\
\hline \multirow{2}{*}{ HISD VU/THD [9] } & Off-line & $98 \mathrm{~ms}$ & Not tripping & Not tripping & $132 \mathrm{~ms}$ \\
\cline { 2 - 7 } & Real-Time & $138 \mathrm{~ms}$ & Not tripping & Not tripping & No
\end{tabular}

\section{Conclusions}

This work introduces a distributed island event detection algorithm based on low-level electrical measurements and avoids the dependency on communication lines, eliminating problems such as packet losses and communications delays. The proposed LISD algorithm can be implemented locally and operate in parallel with the primary controllers, achieving a decentralized island detection system without introducing electrical disturbances typically found in active detection methods. The validation of the proposed algorithm was achieved through an RT simulation environment in a hardware-in-the-loop setting. Overall, the LISD was faster than other reported techniques in the literature (about 3 times) and did not exhibit false-positive tripping in any case. However, the tuning of the required thresholds remains an open problem and sets future research directions. 
Author Contributions: Conceptualization, J.R.L., L.I. and P.P.; methodology, J.R.L., L.I. and P.P.; validation, J.R.L. and P.P.; investigation, J.R.L.; writing—original draft preparation, J.R.L.; writingreview and editing, J.R.L., L.I. and P.P.; visualization, J.R.L.; supervision, L.I., P.P. and A.M.; project administration, P.P. and A.M.; funding acquisition, A.M. All authors have read and agreed to the published version of the manuscript.

Funding: This research received no external funding.

Institutional Review Board Statement: Not applicable.

Informed Consent Statement: Not applicable.

Data Availability Statement: Not Applicable, the study does not report any data.

Conflicts of Interest: The authors declare no conflict of interest.

Abbreviations
The following abbreviations are used
DGU

\section{References}

1. Kroposki, B.; Lasseter, R.; Ise, T.; Morozumi, S.; Papathanassiou, S.; Hatziargyriou, N. Making microgrids work. IEEE Power Energy Mag. 2008, 6, 40-53. [CrossRef]

2. Olivares, D.E.; Mehrizi-Sani, A.; Etemadi, A.H.; Canizares, C.A.; Iravani, R.; Kazerani, M.; Hajimiragha, A.H.; Gomis-Bellmunt, O.; Saeedifard, M.; Palma-Behnke, R.; et al. Trends in Microgrid Control. IEEE Trans. Smart Grid 2014, 5, 1905-1919. [CrossRef]

3. Majumder, R. Some Aspects of Stability in Microgrids. IEEE Trans. Power Syst. 2013, 28, 3243-3252. [CrossRef]

4. Islam, M.M.; Nagrial, M.; Rizk, J.; Hellany, A. General Aspects, Islanding Detection, and Energy Management in Microgrids: A Review. Sustainability 2021, 13, 9301. [CrossRef]

5. Pouryekta, A.; Ramachandaramurthy, V.K.; Mithulananthan, N.; Arulampalam, A. Islanding Detection and Enhancement of Microgrid Performanceback. IEEE Syst. J. 2018, 12, 3131-3141. [CrossRef]

6. Wasowski, M.; Sikorski, T.; Wisniewski, G.; Kostyla, P.; Szymanda, J.; Habrych, M.; Gornicki, L.; Sokol, J.; Jurczyk, M. The Impact of Supply Voltage Waveform Distortion on Non-Intentional Emission in the Frequency Range 2-150 kHz: An Experimental Study with Power-Line Communication and Selected End-User Equipment. Energies 2021, 14, 777. [CrossRef]

7. Arefin, A.A.; Hasan, K.N.B.M.; Othman, M.L.; Romlie, M.F.; Saad, N.; Nor, N.B.M.; Abdullah, M.F. A Novel Island Detection Threshold Setting Using Phasor Measurement Unit Voltage Angle in a Distribution Network. Energies 2021, 14, 4877. [CrossRef]

8. Murugesan, S.; Murali, V.; Daniel, S.A. Hybrid Analyzing Technique for Active Islanding Detection Based on d-Axis Current Injection. IEEE Syst. J. 2018, 12, 3608-3617. [CrossRef]

9. Wang, G.; Gao, F.; Liu, J.; Li, Q.; Zhao, Y. Design Consideration and Performance Analysis of a Hybrid Islanding Detection Method Combining Voltage Unbalance/Total Harmonic Distortion and Bilateral Reactive Power Variation. CPSS Trans. Power Electron. Appl. 2020, 5, 86-100. [CrossRef]

10. Subramanian, K.; Loganathan, A.K. Islanding Detection Using a Micro-Synchrophasor for Distribution Systems with Distributed Generation. Energies 2020, 13, 5180. [CrossRef]

11. Abd-Elkader, A.G.; Saleh, S.M.; Magdi Eiteba, M. A passive islanding detection strategy for multi-distributed generations. Int. J. Electr. Power Energy Syst. 2018, 99, 146-155. [CrossRef]

12. Gupta, P.; Bhatia, R.S.; Jain, D.K. Active ROCOF Relay for Islanding Detection. IEEE Trans. Power Deliv. 2017, 32, 420-429. [CrossRef]

13. Bakhshi-Jafarabadi, R.; Popov, M. Hybrid Islanding Detection Method of Photovoltaic-Based Microgrid Using Reference Current Disturbance. Energies 2021, 14, 1390. [CrossRef]

14. IEEE Standard for Interconnecting Distributed Resources with Electric Power Systems; IEEE Std 1547-2003; 2003; pp. 1-28. Available online: https:/ /ieeexplore.ieee.org/document/1225051 (accessed on 29 October 2021). [CrossRef]

15. IEEE Recommended Practice for Utility Interface of Photovoltaic (PV) Systems; IEEE Std 929-2000; 2000. Available online: https:/ / ieeexplore.ieee.org/document/836389 (accessed on 29 October 2021). [CrossRef] 
16. Balaguer, I.J.; Lei, Q.; Yang, S.; Supatti, U.; Peng, F.Z. Control for Grid-Connected and Intentional Islanding Operations of Distributed Power Generation. IEEE Trans. Ind. Electron. 2011, 58, 147-157. [CrossRef]

17. Jang, S.I.; Kim, K.H. An Islanding Detection Method for Distributed Generations Using Voltage Unbalance and Total Harmonic Distortion of Current. IEEE Trans. Power Deliv. 2004, 19, 745-752. [CrossRef]

18. Ebrahim, M.A.; Wadie, F.; Abd-Allah, M.A. An Algorithm for Detection of Fault, Islanding, and Power Swings in DG-Equipped Radial Distribution Networks. IEEE Syst. J. 2020, 14, 3893-3903. [CrossRef]

19. Lopes, L.; Sun, H. Performance Assessment of Active Frequency Drifting Islanding Detection Methods. IEEE Trans. Energy Convers. 2006, 21, 171-180. [CrossRef]

20. Bakhshi-Jafarabadi, R.; Sadeh, J.; Popov, M. Maximum Power Point Tracking Injection Method for Islanding Detection of Grid-Connected Photovoltaic Systems in Microgrid. IEEE Trans. Power Deliv. 2021, 36, 168-179. [CrossRef]

21. Golestan, S.; Guerrero, J.M.; Abusorrah, A.M. MAF-PLL With Phase-Lead Compensator. IEEE Trans. Ind. Electron. 2015, 62, 3691-3695. [CrossRef]

22. Rocabert, J.; Luna, A.; Blaabjerg, F.; Rodríguez, P. Control of Power Converters in AC Microgrids. IEEE Trans. Power Electron. 2012, 27, 4734-4749. [CrossRef]

23. Khodaparastan, M.; Vahedi, H.; Khazaeli, F.; Oraee, H. A Novel Hybrid Islanding Detection Method for Inverter-Based DGs Using SFS and ROCOF. IEEE Trans. Power Deliv. 2017, 32, 2162-2170. [CrossRef]

24. Hoke, A.F.; Nelson, A.; Chakraborty, S.; Bell, F.; McCarty, M. An Islanding Detection Test Platform for Multi-Inverter Islands Using Power HIL. IEEE Trans. Ind. Electron. 2018, 65, 7944-7953. [CrossRef]

25. OP5700 System Description-Hardware Products Documentation-Wiki OPAL—OPAL RT. Available online: https://wiki.opalrt.com/display/HDGD/OP5700+System+Description (accessed on 15 October 2021)

26. Rapid prototyping controller for power electronics-Control-B Box RCP-Imperix. Available online: https://imperix.com/ products / control/rapid-prototyping-controller/ (accessed on 15 October 2021) 